\title{
Derechos Humanos - Juventud - Políticas Públicas. Un dilema latinoamericano y uruguayo aún por resolver
}

\section{Alejandro Klein Caballero ${ }^{1}$}

\section{Resumen}

El objetivo de este trabajo es generar algunas reflexiones sobre el campo de las políticas públicas dirigidas a la juventud en Latinoamérica y especialmente Uruguay. Su método se centra en una indagación de las fuentes relacionadas al tema con un análisis comparativo crítico de datos, a los que se ha agregado reflexiones sobre los vaivenes entre Estado de bienestar y neoliberalismo y algunas características psicosociales que surgen de los mismos. Entre los resultados obtenidos destaca la necesidad de revisar a profundidad el concepto de "juventud" y "adolescencia" atravesado por un imaginario social o idealizante o denigrante que no permite definir adecuadamente el "sujeto" social hacia el que se dirige estas políticas públicas.

Se indica, como un factor relevante de las consideraciones finales que en tal sentido es necesario incluir variables demográficas, de género, procesos históricos, nuevos tipos de cultura emergente (como las tribus urbanas), las diferencias rurales-urbanas y las transiciones familiares, entre otras, para redefinir certeramente este "sujeto" social y alcanzar entonces un redimensionamento eficaz de las políticas públicas.

Palabras-clave: políticas públicas, estado, juventud, neoliberalismo.

\section{Introducción}

A los efectos de este trabajo no se abordará en extenso la cuestión de qué es ser joven. Es decir, se tomará como joven lo que las encuestas y datos socio-demográficos engloban como tal, aunque intentando introducir algunos elementos de debate en torno al tema. Esta definición de lo joven, tal como se percibe rápidamente, se relaciona directamente a lo etario, ubicándose esta etapa aproximadamente entre los 15 y los 24 años (definición de las OMS). La cuestión no es secundaria pues, como se indicará más adelante, aunque los programas sociales de juventud estén señalados como "para jóvenes”, están destinados no pocas veces a anular o erradicar lo que se considera "perjudicable" de, o en, estos jóvenes -deserción estudiantil, embarazo adolescente, desempleo-.

Los programas dirigidos a los jóvenes - especialmente los que responden a una tradición más claramente neoliberal-, aparecen perfilados explícita o implícitamente para

\footnotetext{
${ }^{1}$ Profesor Investigador de la Universidad de Guanajuato. Associate Fellow- Oxford Institute of Population Ageing. Universidad de Guanajuato. México. E-mail: alejandroklein@hotmail.com
} 
desarrollar competencias, responsabilidades y "compromisos" sociales, que poco disimulan su sentido de adaptación social, buscando incitar en el joven rasgos de conductas claramente reproductibles y controlables. Estos énfasis en las políticas de juventud tanto en América Latina como en Uruguay, son los que pretenden discutirse en este trabajo. En la primera parte, se presentan algunos elementos generales sobre la juventud latinoamericana, para luego puntualizar sobre la juventud en Uruguay. En la segunda parte, se discuten las rupturas y continuidades en las políticas dirigidas a este grupo poblacional, y finalmente, se presenta una reflexión sobre los retos más importantes en este campo.

\section{Juventud en Latinoamérica: algunos datos preocupantes}

Para el año 2000, se estimaba la existencia de 1,066 millones de jóvenes en el mundo, concentrándose el $60 \%$ de ellos en Asia, el 16\% en África y 10\% en América Latina y el Caribe. La franja de juventud para entonces alcanzaba casi el $20 \%$ del total de la población de América Latina, porcentaje que tiende a decrecer, ya que las proyecciones para el año 2010 indicaban que los jóvenes llegarían a representarían el 17.8\% de la población (CEPAL, 2001). Casi el $80 \%$ de este grupo poblacional vive en zonas urbanas, y solo el $20 \%$ restante lo hace en zonas rurales. Alrededor del $50 \%$ vive en situación de pobreza (tomando el margen de edad 13 a 19 años), lo que representa alrededor de 114.2 millones de personas. Además, entre 1994 y 1999, el nivel de desocupación entre los jóvenes creció del 14\% al 20\% en la región (CEPAL/UNICEF/SECIB, 2001).

Otros datos igualmente preocupantes indicaban que para ese mismo año, cerca de la mitad de los jóvenes urbanos de 20 años de edad habían desertado total o parcialmente del sistema educativo. En las zonas rurales la situación era aún más crítica: casi tres de cada cuatro jóvenes se encontraban en esa situación. Asimismo, alrededor de un 15\% de las jóvenes latinoamericanas han sido madres antes de los 18 años, mientras que un $16 \%$ del conjunto de migrantes interregionales corresponde a la franja joven. Se puede perfilar al joven latinoamericano entonces, como mayoritariamente urbano, predominantemente pobre y especialmente afectado por el desempleo y las dificultades en el acceso a las oportunidades sociales, educativas y culturales. El diagnóstico en esta materia da cuenta de evidentes problemas de exclusión, marginación de oportunidades sociales y cronicidad de problemas estructurales que las políticas sociales no han podido resolver satisfactoriamente.

Es necesario indicar sin embargo, que no es posible realizar un diagnóstico homogéneo de esta población en la región, pues no es la misma situación la que padecen 
jóvenes provenientes de familias de bajos ingresos, que los que provienen de familias con ingresos altos. En el caso de Brasil, por ejemplo, “en 1990 el 19.4\% de los jóvenes urbanos entre 13 a 17 años, de hogares ubicados en el cuartil más bajo de ingresos, no trabajaba ni estudiaba; mientras el porcentaje correspondiente al cuartil más alto era de 3.6\%.6" (CEPALCELADE, 2000: 173). Igual situación se verifica en el caso de la fecundidad: "el grueso de la reproducción biológica se concentra en las jóvenes de los segmentos más pobres de la población, en tanto la fecundidad de las jóvenes de los sectores medios y altos ha bajado sistemáticamente a lo largo del tiempo" (CEPAL- CELADE, 2000: 174).

Estas características están íntimamente vinculadas a lo que se ha denominado la reproducción intergeneracional de la pobreza (CEPAL, 1994). Es destacable además, cómo el desempleo juvenil no se relaciona solo con la falta de destrezas laborales, sino también con la continua elevación del nivel de exigencias para adquirir un trabajo. En el año 2000 se estimaba una media necesaria de 12 años de preparación escolar (CEPAL-CELADE, 2000). Conseguir un empleo se hace cada vez más difícil tanto como conservarlo, todo lo cual genera sentimientos de inseguridad y desconcierto (Klein, 2006). Habría que indicar también que el homicidio se ubica entre las principales causas de muerte entre los jóvenes. En Brasil por ejemplo, es la principal causa de muerte de este grupo, correspondiéndole un $25.3 \%$ del total de muertes registradas (UNICEF/IBGE, 1997).

En el caso de la educación se estima que la tasa de deserción de los adolescentes y jóvenes del $25 \%$ de hogares de más bajo ingreso, es tres veces mayor que la del $25 \%$ de hogares de ingreso mayor. Para el año 2000, cerca de 15 millones de jóvenes de entre 15 y 19 años de edad habían abandonado la escuela antes de completar los 12 años de estudio que actualmente se consideran el nivel básico. Alrededor del 70\% de ellos (10,5 millones) ya lo habían hecho antes de poder completar la educación primaria (CEPAL, 2002). Pero además de los procesos de deserción escolar de las clases pobres, habría que destacar la progresiva privatización de la educación en los sectores medios y altos (CEPAL-CELADE, 2000), con la consecuencia de que la educación pública aparece devaluada, "desfasada" y por extensión poco motivante, dado que ya no es suficiente para generar las condiciones de ingreso al mundo laboral (Klein, 2006).

El informe sobre Panorama social de América Latina (1998), indica que durante el período 1990-1997, la problemática del desempleo recaía substancialmente en los grupos sociales de menos recursos. Los jóvenes de 15 a 24 años que no estudian ni trabajan, representan entre el $12 \%$ y el $40 \%$ en los hogares pobres y entre el $2 \%$ y el $10 \%$ en los hogares 
de más elevados ingresos (CEPAL-CELADE, 2000). En mayor o menor medida, los datos precedentes indican que la juventud latinoamericana se ha visto especialmente afectada por algunos procesos sociales y culturales introducidos por el neoliberalismo ${ }^{2}$. Este grupo aparece estratificado socialmente, perdiéndose probablemente puntos de contacto o áreas en común dentro de las diversas modalidades de la experiencia de ser joven. Pero este panorama no se limita solo al mundo laboral y estudiantil, también transversaliza el universo adolescente en general:

Las jóvenes más pobres acumulan un número de hijos(as) significativamente mayor que el promedio de sus países y muy superior al de las jóvenes del quintil socioeconómico más alto...esta diferencia implica que las jóvenes de los estratos altos tienen una carga de crianza muy inferior a la de sus homólogas de los estratos bajos, hecho que las dota de más tiempo y opciones para la acumulación de activos o la realización individual (CEPAL-CELADE: 2000, 36).

Más que de homogeneidad de situaciones, habría que hablar de "coexistencia" o "conflicto" de diversas experiencias y marcos sociales donde las oportunidades, el tipo de formación y las inserciones laborales dependen menos de lo "joven” y más de estructuras sociales diferentes y hasta contrastantes:

En los diferentes grupos de población joven es habitual que no compartan beneficios, ni metas, ni futuro. Casi se podría agregar que no comparten el sentido de pertenencia a la misma sociedad, porque evidentemente no se trata de "la misma" sociedad" (CEPAL, 2003:15)

Se hace pues cada vez más difícil hablar de "juventud" en general sin referencia a particularidades sociales y culturales. Un caso especial es el de los jóvenes de origen indígena, donde además de las diferencias sociales, se suman diferencias raciales discriminatorias (CEPAL, 2003).

\section{Aquél Uruguay que fue}

Uruguay supo conocer días de gloria en los que la sociedad de bienestar se inscribía con letras mayúsculas en su cuerpo social y en su organización estatal. La ciudadanía era una forma de cultura que los uruguayos alentaban, reconociéndose en esa matriz compartida. Se verificaba así la concreción de un Estado 'generoso' y abarcador que se expresaba claramente

\footnotetext{
${ }^{2}$ Para ampliar esta discusión se pueden consultar O’Donell (1999), Forrester (2000) y Sader y Gentili (1999).
} 
en los hechos y en los discursos que recorrieron al Uruguay batllista ${ }^{3}$ y neo-batllista, desde principios de siglo veinte hasta fines de la década de los cincuenta (Nahum, 1975; Bértola, 2000; Barrán-Nahum,1985). Ese batllismo dentro de la mejor tradición keynesiana, constituyó -y expresó- un discurso de tipo integrador, que supuso: "la articulación de un sistema de relaciones diferenciales que pretendían integrar selectivamente las demandas populares a la hegemonía expansiva del bloque en el poder" (Panizza, 1990: 16). Este discurso integrador ${ }^{4}$ impulsó un modelo de ciudadanía que condensó "una variedad de elementos políticos, sociales y económicos; identidades alternativas en términos de clase, religión y pertenencias regionales que fueron durante mucho tiempo un 'impensable' para la mayoría de la población" (ídem: 36).

De esta manera, y aún dentro del capitalismo periférico, el estado benefactor supo ser parte de la identidad económica y social del Uruguay. Se configuró así una sociedad amplia e inclusiva (Klein, 2006), donde la identidad del sujeto se moldeaba de acuerdo al patrón de ciudadanía, potenciándose el par derechos- obligaciones como una situación que enriquecía el "cuerpo" social, tanto como el espacio individual. La clase media, que sin duda recibió los diversos beneficios del Estado, se consolidó como sinónimo de trabajo, honradez, modelo de la sociedad uruguaya y meta posible a ser alcanzada:

El Estado Benefactor de principios de siglo XX configuró dentro del proyecto modernizador, la extensión de una clase media gracias a la generación de una fuente de trabajo que, aunque no colmara la totalidad de las posiciones, sí homogeneizaba centralizando la imagen de solidez y orden sobre sí para la extensión de toda la sociedad.(....) Y es que la clase media uruguaya desborda toda identidad de clase, está instalada en el imaginario social como la forma misma del ser social, extendiéndose hacia arriba y hacia abajo en valores y normas de conducta en los cuales lo único compartido es el horizonte que enmarca a las subjetividades (Araujo: 2002, 70-71).

Las transformaciones dentro de clase media uruguaya, sus vicisitudes, formas de presentación social y sus construcciones subjetivas, dan cuenta, no solo de la modernidad keynesiana, sino también de los cambios que ha padecido el Uruguay desde los formatos neoliberales (Klein, 2006 y Astori, 1989).

\footnotetext{
${ }^{3}$ Refiere a José Batlle y Ordoñez, presidente de Uruguay a comienzos del siglo XX (1901-1904 y 19121916) y fundador del "batllismo" fuerza política modernista y progresista.

${ }^{4}$ Es de hacer notar que esta situación es inseparable de la pregnancia de los partidos políticos en Uruguay, lo que me es imposible profundizar por escapar al marco de este trabajo.
} 


\section{EI Uruguay que es: jóvenes en los contextos contemporáneos}

Uruguay se caracteriza por tener una pirámide poblacional envejecida, con baja tasa de nacimientos y escaso número de hijos por familia. El número de adolescentes representa aproximadamente el 16,5 \% del total de la población. Esta baja población juvenil se explica también, en parte, por la fuerte corriente migratoria hacia diferentes países que se verifica desde los años sesenta y que afecta fundamentalmente a los varones. La mitad de los jóvenes residen en la capital del país: Montevideo (CELADE ,1998). Este grupo ha sido uno de los más afectados por la pobreza. Según el Instituto Nacional de Estadística de este país, en 2004, esta se concentraba significativamente en los sectores poblacionales jóvenes:

En 2004 , el $32 \%$ de las personas del país eran pobres, entre estas el $57 \%$ tenía menos de 6 años, el 54\% entre 6 y 12, el $45 \%$ entre 13 y 17, el 29\% de adultos y el $11 \%$ de personas en la tercera edad. De las 108.200 personas indigentes del año 2004, se estiman en 47.700 los adultos (entre $41.900 \mathrm{y}$ $53.600)$ y en 60.500 los menores de 18 años (entre 52.900 y 68.000 )" (Instituto Nacional de Estadística, 2004: 18).

En materia de empleo, este grupo representa uno de los sectores más golpeados por el desempleo. Los jóvenes de entre 14 y 29 años, constituyen el 63\% de los desempleados del país, provenientes a su vez de familias de más bajos ingresos (Cinterfor, 1995). Es posible además indicar que aún ingresando al mundo del trabajo, los jóvenes no logran contratos estables y a largo plazo, y reciben además, menores salarios que otros grupos etarios (Ministerio de Educación y Cultura, 1998). Los problemas de exclusión también se manifiestan en el sistema educativo. Estudios realizados en el ámbito de la ANEP (Administración Nacional de Enseñanza), afirman que la deserción en el nivel secundario ocurre mayoritariamente en los jóvenes y adolescentes pertenecientes a los hogares de más bajos ingresos (ANEP, 2002). Sin embargo, comparado con otros países de América Latina, Uruguay se presenta como un país con baja deserción temprana (antes de completar el ciclo primario):

Pero a medida que son tenidos en cuenta niveles superiores de educación, la deserción aumenta significativamente y el posicionamiento de Uruguay comparado con otros países de América Latina cae en forma abrupta. En Uruguay, el $44 \%$ de los varones de 15 años de edad y bajos ingresos (primer quintil) se encuentra fuera del sistema educativo, en cuanto esta cifra cae para Chile en 18,3\% y en Argentina 21,5\%, prácticamente la mitad que para Uruguay (ídem: 24). 
Otros estudios como los de Furtado (2003), confirman los datos anteriores y señalan que Uruguay comparte con México la peor ubicación de un ranking de 18 países latinoamericanos en cuanto a la deserción estudiantil. Además, es el país con mayor deserción asociada a la pobreza. Mientras que para América Latina el 43\% de los desertores proviene del primer cuartil de ingresos, en Uruguay esa cifra alcanza al 63\% .

\section{La juventud uruguaya y latinoamericana: ¿Rupturas de un contrato social?}

Los datos sugieren un cuestionamiento radical al contrato implícito entre la adolescencia y las posibilidades de trabajo y educación ofrecidas por el estado benefactor (Klein, 2006). Este contrato enunciaba de forma tácita, pero no por eso menos vigorosa, que la sociedad es un espacio amplio y generoso, con capacidad de "albergar" a un nuevo miembro en ella a través de la formulación de derechos y obligaciones, que incluían perspectivas laborales y/o de estudio: "Trabajo y educación fueron prácticas privilegiadas para dar sentido a la identidad en la modernidad. Sin embargo, el proceso histórico pone de manifiesto la crisis de estos modos de socialización" (Cohendoz, 1999: 2). A cambio, se esperaba que el sujeto cumpliera ciertos requisitos imprescindibles en relación a crecimiento, madurez y experiencia, lo que lo habilitaría a ser nombrado y auto-nombrarse como un ser adulto-ciudadano. Sin embargo las cosas se han modificado. Desde la lógica neoliberal parece que la sociedad ya no alberga sino que desampara. Ya no tiene (o así se intenta imponer desde el imaginario) lugar para todos, y el pasaje por distintas experiencias etarias se torna angustiante:

Sobre o pano de fundo de uma relativa descronologização do percurso das idades, e uma dificuldade geral de lograr inclusão plena, a entrada no mundo adulto se faz cada vez mais tarde (estendendo ainda mais o tempo da juventude), segundo etapas variadas e desreguladas (Abramo, 2005: 44).

De esta manera, entre los grupos sociales más afectados por el neoliberalismo se encuentra la juventud empobrecida. La moratoria tradicionalmente adscripta al universo joven como facilitadora en el proceso de inserción social, entra (valga la redundancia) en moratoria desde la gestión neoliberal (Klein, 2006). El joven si se prepara para algo, tal como indican los datos precedentes, es para la inactividad, la emigración, la violencia, la exclusión o el desaliento:

Con la gradual implantación de medidas neoliberales asistimos a una vehemente producción de inseguridad, miedo y pánico, articulados al crecimiento del desempleo, la exclusión, la pobreza y la miseria. Los jóvenes pobres, cuando escapan del exterminio, son los 'excluidos por excelencia', pues ni siquiera consiguen llegar al mercado de trabajo formal. Para los que consiguen sobrevivir, están previstos diferentes tipos de 
encarcelamiento. Muchos jóvenes pobres mayores de 18 años están confinados en las prisiones (Fraga, 2003: 27-28) .

No es sorprendente percibir que las instituciones que antaño incluían e integraban un "joven conductor de acción dirigido al futuro" (Fraga, 2003: 46), se encargan hoy por el contrario, de eliminar las condiciones y posibilidades de su integración. Aunque el joven consiga un trabajo es factible que, o quede rápidamente desempleado, o que ocupe cargos de rango netamente inferior (Tavares, 1999; Fraga, 2003). De esta manera, la situación de escepticismo que mantienen los jóvenes, en el fondo, parece ser bastante realista si consideramos las denigrantes ofertas laborales dedicadas a este grupo, los bajos salarios y la inestabilidad de sus contratos de trabajo. Igualmente, en muchos casos, la educación se transforma en causa de exclusión (Abramovay, 2002).

Esta situación consolida un sentimiento de desencanto generalizado por lo que "El discurso de los jóvenes está permeado, casi todo el tiempo, por el miedo al futuro" (Fraga, 2003: 259). Progresivamente, enormes grupos de jóvenes son marginados de los sistemas de enseñanza, del trabajo y en general de los derechos de ciudadanía. Esta falta de expectativas y de "escasez" de derechos implica la imposibilidad de seguir sosteniendo una "prometida" inserción social (Klein, 2006). Se hacen presentes aspectos de un proceso de desciudadanización que se agudiza obviamente entre los grupos sociales más pobres y desprotegidos, por lo cual el joven pierde marcos de referencia identitarios y de integración social, con extrema dificultad para que emerjan referentes sociales substitutivos.

La promesa del bienestar se debilita en tanto la adolescencia ya no pasa a ser etapa de "integración" social, sino de "extrañamiento" social. Los derechos y las obligaciones ya no pueden establecerse "a priori", debido a que las mismas se formulaban y ajustaban preferentemente a través del trabajo y la educación, los que están ahora cuestionados y relativizados. Es un sujeto que pierde calidad de individuo, si entendemos por "individuo" una construcción social- subjetiva inseparable del marco de los derechos sociales (Klein, 2006). Cabe entonces preguntarse ¿cuál es el lugar social que pasan a ocupar los jóvenes si los procesos tradicionales de integración social se debilitaron?. Una hipótesis refiere la aparición de rituales de expiación y sacrificio:

A época moderna está assim vendo se disseminar uma nova forma sacrificial: o sacrifício ao quadrado. Trata-se de um gesto sacrificial novo que, ao se perpetrar, permite criar o ponto de apoio necessário, que faltava, 
para enfim viver, nem que seja por um instante, antes de desaparecer. Essa nova forma sacrificial começa com o sacrifício de vítimas precisamente escolhidas (...) Com certeza resta um apelo desesperado ao laço social” (Dofour, 2005: 102).

Surgen así, estructuras sociales y culturales regresivas donde se consolida un ritual del sacrificio impuesto a jóvenes que mueren simbólica o violentamente. Dentro de esta situación también se resitúa el debate sobre la problemática de la "vulnerabilidad social” y la violencia social entrelazada a la violencia familiar:

Muchos de los jóvenes [que sufrieron violencia social] tuvieron contacto con la violencia de forma directa también en el ambiente familiar... muchos de los niños encontrados en las calles dejaron a sus familias por ser víctimas de malos tratos por sus propios padres" (Abramovay, 2002: 50).

\title{
Políticas Públicas dirigidas a la Juventud: el desafío latinoamericano entre la tradición y el cambio
}

Como es sabido muchas de las reformas políticas y sociales llevadas a cabo en América Latina en los años ochenta y noventa, fueron impulsadas al amparo del "Consenso de Washington" y giraron alrededor de: "asuntos de disciplina fiscal, liberalización de la política comercial y del régimen de inversiones, desregulación de los mercados internos y privatización de empresas públicas, con resultados escasos” (Rodríguez, 2003:11). Un rasgo importante dentro de estas políticas públicas durante la gestión neoliberal, fue el pasaje de la figura del ciudadano recibiendo un derecho, a la de un cliente o usuario usufructuando un servicio. Al mismo tiempo, y especialmente en el caso latinoamericano, paralelamente al proceso que Tavares (1999), denominó “descentralización destructiva:

\begin{abstract}
Según la concepción neoliberal de política social, el bienestar social pertenece al ámbito privado. Este proceso causado por políticas deliberadas de ajuste y no por una 'fatalidad global' es uno de los principales mecanismos generadores de exclusión hoy en Brasil y América Latina (idem: 181)
\end{abstract}

Para la autora, esto ha generado: "un sinnúmero de nuevas formas de exclusión social, en la medida, en que empeoraron las condiciones de empleo y trabajo [lo que] creó un desempleo (...) seguramente el costo social más alto” (idem: 174-175). El costo social que ha dejado el neoliberalismo ha ampliado las brechas sociales y evidenciado la necesidad de generar nuevas 
acciones para este sector poblacional. En mayor o menor medida todos los países de la Región han implementado -y aún desde el período neoliberal- programas de acción, o políticas nacionales de juventud, o mecanismos de coordinación nacional para asuntos de juventud y en general programas de acción nacional para la juventud (ONU, 2001). Organismos trasnacionales como la Organización Iberoamericana de Juventud y otros han participado en estas iniciativas. Desde 1992 se creó la Organización Iberoamericana de Juventud (OIJ), cuya dinámica de trabajo se enmarca dentro de las líneas orientadoras aprobadas por las Conferencias Iberoamericanas respectivas.

Desde el año 2000, la OIJ mantiene los siguientes ejes programáticos: formulación de la Carta Iberoamericana de la Juventud; desarrollo de la Comisión Internacional por los Derechos de la Juventud y la Adolescencia; articulación de un marco de orientación para guiar las políticas de juventud basado en la noción de ciudadanía integral; evaluación de las políticas de juventud en Iberoamérica; fortalecimiento y modernización de los organismos oficiales de juventud, entre otras iniciativas (Bango, 1999). En el informe de la OIJ: "Políticas de juventud en América Latina: evaluación y reformulación”, se realizó una evaluación de lo que puede ser considerado el modelo "tradicional" de las política públicas dirigidas a la juventud. Las políticas educativas, recreación y de uso del tiempo libre aparecen como los enfoques predominantes durante el período 1950-1980 (Rodríguez, 2008). Hay que destacar que los jóvenes a quienes se dirigieron estos programas se han caracterizado en su mayoría por ser pobres, desempleados y con bajo nivel educativo, aspectos que los clasifican dentro de factores de "riesgo social" y por tanto sujetos de las políticas y acciones públicas.

Otro sector de políticas públicas se ha dirigido a los jóvenes considerados "excluidos", tradicionalmente de origen urbano y con énfasis en su inserción al mercado laboral, en la necesidad de generar "habilidades sociales", orientación profesional, fomento de la integración grupal y mejoramiento de la autoestima. Por último, habría que señalar las políticas de salud enfocadas en la prevención del consumo de drogas, prevención del SIDA y del embarazo precoz. Puede pensarse que el objetivo más o menos velado de estas iniciativas implica la erradicación del peligro de la "vagancia", la "irresponsabilidad" y el "desenfreno", como productos que prejuiciosamente se considera, podrían surgir si no se mantiene una actitud "atenta" y vigilante sobre los jóvenes. El mejor ejemplo de esto, es el enfoque "sanitarista" que revela una preocupación por lo "anómalo", lo "extraviado", por lo que sale de una norma fuertemente normativizada. Se ocupa entonces de jóvenes integrados o “integrables” (Rodríguez, 2008). 
Un efecto indeseado de este tipo de enfoques que han predominado en muchas de las políticas para jóvenes, es que acaban por generar una imagen negativa de la juventud, asociándola a lo problemático y a lo conflictivo, desde una perspectiva estereotipada y estigmatizante. Uno de estos aspectos se refiere quizás a cierta tendencia psicologizante que puede llegar a ser fuertemente culpabilizante. Es decir, por tomar un ejemplo, se supone que si el joven no adquiere trabajo es por falta de herramientas adecuadas, las que una vez proporcionadas, deberían llevar a resolver dichas limitaciones, lo que significa que el problema de la inserción al mundo laboral se transformara en algo personal y privado. En este punto, si el joven no adquiere un trabajo es porque no quiere o no le interesa obtenerlo, lo que en otras palabras significa que este pasa a ser el "culpable" de su propio desempleo. Este enfoque olvida que la situación es mucho más compleja en relación a factores sociales y públicos que dificultan el acceso al mundo del trabajo, más allá de la voluntad del sujeto en cuestión.

Probablemente la insistencia de las políticas públicas tradicionales implica una visión institucionalista de la juventud (CEPAL, 2003), la cual busca otorgar a los jóvenes herramientas educativas y laborales que difícilmente, sin embargo, se correlacionan con lo que es el estudio y el trabajo en la sociedad actual:

\begin{abstract}
El modelo de políticas de juventud centrado en la educación y el tiempo libre, vigente a lo largo de casi todo el siglo XX, fue un enfoque adulto, conservador y funcionalista, en el más estricto sentido de los tres términos utilizados, en la medida en que se toma a la sociedad vigente como un dato que no se cuestiona, y se define como objetivo específico en relación a las generaciones jóvenes, su integración futura a dicha sociedad, de modo tal que la reproducción de la misma en el tiempo y en el espacio esté asegurada. Dicho enfoque, como se sabe, funcionó con cierta fluidez en el marco de economías expansivas y dinámicas, que aseguraban cierta "movilidad social ascendente", sobre todo para los jóvenes "integrados", pero mostró serias limitaciones para responder a la problemática de los jóvenes "excluidos", especialmente en las etapas de crisis (Rodríguez, 2003:14)
\end{abstract}

Otro factor de especial dificultad se relaciona con que estos programas no están insertos en una política nacional de juventud y tienen generalmente un enfoque sectorial, teniendo diagnósticos poco precisos y escasas evaluaciones sobre grado de efectividad e impacto. De esta manera entran en conflictos con otras reparticiones estatales sin posibilidad de generar políticas de articulación y enlace. 
Algunas de las evaluaciones sobre este tipo de programas, indican la necesidad de incorporar variables sociales, manteniendo una perspectiva interdisciplinaria y buscando además evitar situaciones de dependencia o "asistencialismo", estimulando a la vez, la participación decisiva de los jóvenes, no solamente en la ejecución de los programas, sino además en su proceso de elaboración (Pilotti-Camacho, 2003). El desafío pasa entonces por la formulación de políticas públicas más realistas, apoyadas en estrategias más adecuadas para: "asegurar que las acciones sean más pertinentes y aplicables" (CEPAL-CELADE: 2000, $60)$.

Sin embargo, y pese a los retos en esta materia, tampoco es posible afirmar que el panorama es totalmente negativo. Por el contrario, hay indicadores que señalan éxitos en algunas áreas. Los programas de prevención y tratamiento del consumo de drogas, los programas de prevención y atención de las enfermedades de transmisión sexual y los programas de prevención de los embarazos registran avances significativos. Lo mismo podría decirse de algunas iniciativas enfocadas en la inserción laboral:

Las evaluaciones realizadas subrayan que algunos aspectos de la focalización han funcionado bien, tanto en términos sociales como de género. Los jóvenes que participaron en estos programas disfrutan de ventajas que no están al alcance de aquellos que no lo han hecho: disponen de mayores facilidades para su inserción laboral, empleos más estables, condiciones más apropiadas de trabajo y mejores relaciones sociales (Rodríguez, 2003:4).

Probablemente el punto más positivo se relaciona con que desde finales de los años noventa, se ha venido consolidando un formato de las políticas públicas centrado en la necesidad de generar espacios de efectiva participación juvenil, además, de poner en discusión la necesidad de incorporar otros modelos de desarrollo en torno a los jóvenes. Puede pensarse que hay un reposicionamiento de la agenda y el debate social frente al fracaso evidente del mercado por regular de forma equitativa las inequidades sociales (Sader-Gentili, 1999). Este principio de renovación de ciudadanía se anuda a una vigorosa revalorización de la cultura cívica, con el consiguiente reposicionamiento de las políticas sociales.

Los desafíos en esta materia, implican el abandono de posturas conservadoras y funcionales por otras de cambio y transformación (Rodríguez, 2008), logrando una efectiva articulación entre los diferentes organismos enfocados al tema juventud, transversalizando o descentralizando la temática de juventud más que centralizándola en un organismo específico 
para jóvenes, operatoria que más allá de sus créditos políticos no ha revelado eficacia. Por otro lado, cabe pensar si es posible trazar un nuevo "mapa" en este tipo de políticas enfocadas en una perspectiva generacional de ciudadanización, que evite sin embargo caer en los espacios y programas exclusivos para jóvenes, que no pocas veces revelan ser una serie de actividades inconexas, con falta de diagnósticos y procesos de evaluación precisos (Rodríguez, 2003, 2008).

\section{Políticas Públicas de Juventud en Uruguay: ¿durmiéndose en los laureles?}

Uruguay en mayor o menor medida, comparte a grandes los rasgos y modelos de las políticas públicas indicadas para la Región. Sin embargo, vale la pena detenerse en algunas características específicas. El Uruguay tradicionalmente se ha destacado en América Latina por su cultura democrática (Canzani, 2000), la cual ha estado claramente asociada a una estructura social menos desigual que la de sus vecinos de la Región (Rossel, 2009). Otro rasgo refiere a una larga tradición en materias de políticas públicas, asentadas en un fuerte ofrecimiento de oportunidades a los sectores menos favorecidos:

En Uruguay las políticas de juventud son instituciones públicas en el sentido que plantea Thwaites desde hace varias décadas. Ya sea por iniciativa de los progresismos $^{5}$ o quienes los precedieron en los gobiernos, es inocultable la relevancia que la sociedad política les atribuye a las problemáticas que esas políticas vienen a resolver (Ambrosi-Sanseviero, 2009 :13)

Este rasgo de continuidad no descarta un creciente panorama de déficits sociales y nuevas formas de desigualdad, inéditas para la forma en cómo Uruguay se ha visto y pensado tradicionalmente. Los cambios además han implicado un nuevo lugar de los jóvenes que han entrado en franca confrontación con generaciones anteriores, debido a que ya no se sienten allegados a las formas organizativas de los partidos políticos, indicando como un hecho insólito para la mentalidad uruguaya, disconformidad con el funcionamiento de la democracia uruguaya (ANEP, 2002). Se trata pues de un nuevo tipo de juventud, desilusionada y escéptica.

\footnotetext{
${ }^{5}$ Progresismo y Gobiernos progresistas refiere a las tres últimas administraciones que ha tenido Uruguay en su gobierno: la de Tabaré Vázquez (2005-2010; 2015-2020) y la de José Mujica (2010-2015).
} 
Una reflexión que solo se esboza, pero que merece un mayor desarrollo, refiere a que si las políticas públicas tradicionales dirigidas a la juventud en Uruguay se han estructurado desde un modelo idealizado de clase media, cómo pensar otras, diseñadas desde el empobrecimiento, que es la característica del Uruguay de hoy. En este sentido, la afirmación: "los jóvenes más empobrecidos de hoy son los que dotarán al país de la mayor parte de su población futura" (Rossell, 2009:73), no refiere solo a un dato socio-económico, sino que implica además al desafío de un modelo de nuevas políticas emergentes que permitan ofrecer nuevas bases de ciudadanía y participación, que sin dejar de lado los referentes del empleo y la educación, incluyan también la problemática joven del escepticismo y la desazón generacional.

\section{Conclusiones}

Como se ha indicado, el panorama de la juventud en la Región es extremadamente heterogéneo, más allá del empobrecimiento "homogeneizador" que ha surgido desde la gestión neoliberal. Esta heterogeneidad se debe no sólo a diferencias sociales, sino también a otras variables como el género, los procesos históricos, los nuevos tipos de cultura emergente (como las tribus urbanas), las diferencias rurales-urbanas y las transiciones familiares, entre otras. Sin embargo las políticas públicas dirigidas a la juventud (tanto las llamadas tradicionales como las de tipo neoliberal) han mantenido un paradigma fuertemente homogeneizador, sosteniendo una concepción de "juventud" resultado más de abstracciones o productos del imaginario social, que de diagnósticos y análisis más fiables. Podría decirse que las políticas públicas surgen muchas veces como uniformizadoras e institucionalizadoras frente a un panorama cultural y subjetivo que plantea sin embargo la dificultad de adscribir variables institucionales a lo que hoy se denomina como joven (Klein, 2010). Por tanto, uno de los retos de las políticas sociales alternativas dirigidas a la juventud, consiste en discutir y reorientar estas tendencias.

Podría decirse, que de alguna manera se ha generado probablemente un desfase entre el "objetivo" de estas políticas sociales y el "sujeto" de las mismas, producto más de enunciados idealizadores o denigradores, que de un diagnóstico situacional e interdisciplinario preciso. Si se tiene en cuenta el conjunto de cambios que se vienen perfilando a nivel social y cultural, se torna relevante analizar si las políticas públicas son capaces de integrar múltiples escenarios con diferentes problemáticas que oscilan entre roles en transición, cambios en la concepción de género, movilidad migratoria, tecnologías 
informáticas y predominancia de nuevas modalidades de ciudadanía (Rodríguez, 2008; Kymlicka -Wayne, 1997).

Las políticas públicas neoliberales heredaron y profundizaron este problema de diagnóstico de lo que es ser joven (en tanto es un diagnóstico que aparece tradicionalmente fuertemente descontextualizado socialmente), substituido generalmente por un imaginario social que oscila entre una imagen idealizada y una imagen negativa de éste -aquel deseoso de entrar al mercado de trabajo, en el primer caso y el joven que presenta tendencias "naturales" hacia la haraganería y la delincuencia, en el segundo, por dar dos ejemplos muy generales-. Pero no se trata solamente de modificar la imagen de lo "joven" y la "juventud", sino además de realizar un análisis crítico de aquellas políticas sociales que siguen interpelando a la "juventud" desde un modelo social que ha entrado en una profunda crisis desde el momento en que ya no se puede garantizar alta estabilidad en oferta de empleos y oportunidad educativa (Klein, 2006).

La situación antes enunciada genera un agudo problema en el campo social, en tanto surge el peligro de que las políticas públicas en vez de generar inclusión, acaben por generar expulsión, pues plantean un modelo de juventud (y correlativamente de sociedad) inalcanzable e idealizado. Por otra parte, es necesario destacar cómo algunas de estas políticas ponen el acento en la eficiencia de la ejecución de los roles adultos tradicionales (como el acceder al mundo del trabajo), mientras que -sin embargo- la propia sociedad genera las condiciones de imposibilidad de tal ejercicio. Por otro lado, se hace cada vez más difícil sostener claros indicadores de diferencia entre el mundo adulto y el mundo joven desde el:

...carácter crecientemente difuso de las fronteras entre el mundo juvenil y el mundo adulto. En el pasado, la entrada al mundo adulto implicaba la convergencia en el tiempo de comportamientos económicos, sociales, culturales y políticos, modelados según patrones de conducta bien establecidos...Actualmente se aprecian al menos dos procesos que modifican esta situación: por un lado, el aumento sostenido de la proporción de personas que participan plenamente en la economía sin dejar por ello de seguir identificándose con una cultura juvenil y, por el otro, las incertidumbres que rodean la inserción laboral y la formación de la familia" (CEPAL-CELADE:2000, 12).

Es posible también indicar otro factor de cambio desde las tendencias sociodemográficas que revelan un sostenido proceso de envejecimiento poblacional, por lo que el número de jóvenes (entre 15 y 29 años) por cada 100 adultos disminuirá de 80 en el año 2000 
a 46 en el año 2050. Pero: "La relación entre jóvenes y adultos mayores (65 años y más) se reducirá de manera incluso más dramática: de 520 jóvenes por cada 100 adultos mayores bajará a 290 en 2020 y sólo a 80 en 2050” (CEPAL-CELADE, 2000). Por tanto, el mundo se hace cada vez "menos joven” y cada vez "más viejo”. Por tanto cabe preguntarse ¿cómo repercutirá esta nueva realidad en la forma en cómo se diseñarán las políticas públicas en las próximas décadas?. Es algo difícil de vaticinar, pero sin duda las mismas recibirán el impacto de una población cada vez más "escasa" y "unario" generacionalmente, en el sentido de que cada vez habrá menos niños y jóvenes, y más población adulta y vieja.

Se ha indicado que una dificultad de las políticas públicas dirigidas a la juventud puede erradicar en la transitoriedad de la misma (Rodríguez, 2008), pero es una observación claramente insuficiente. Se retoma la perspectiva etaria tradicional, pero además olvidando que en definitiva, todos los sujetos de las políticas sociales son "transitorios". Un adulto no es adulto "ad eternum", menos lo es un niño o un anciano. El debate pasa por otro lado y se refiere -punto imposible de desarrollar en este trabajo- a las nuevas formas de construcción de la subjetivad y lo subjetivo.

Al mismo tiempo, asistimos sin duda a un nuevo horizonte en políticas sociales asociadas al llamado posneoliberalismo, en relación al descrédito del neoliberalismo "clásico". Dentro de este panorama emergente, además de una revisión crítica del enfoque tradicional del diagnóstico sobre qué es ser joven, se busca incorporar las variables ya mencionadas: envejecimiento demográfico, nuevas configuraciones familiares $\mathrm{y}$ especialmente las nuevas formas de promoción de ciudadanía, las que se van constituyendo en la plataforma nodular del nuevo enfoque en políticas sociales. Sin duda, un tema candente y desafiante para la región y que tendrá que evaluarse en unos años, para saber que tanto los procesos y formatos de estas nuevas tendencias permiten responder cabalmente hasta dónde se podrá llegar y qué se podrá realizar desde estas nuevas realidades sociales, culturales y subjetivas.

\section{Human Rights-Youth- Public Policies. A Latin American and Uruguayan dilemma yet to be resolved}

\section{Abstract}

The objective of this paper is to generate some reflections on the field of public policies 
aimed at youth in Latin America and especially Uruguay. His method focuses on an investigation of the sources related to the subject with a critical comparative analysis of data, plus some reflection in the swings between welfare state and neoliberalism and some psychosocial characteristics that arise from them. Among the results obtained, it is important to review in depth the concept of "youth" and "adolescence" crossed by a social or idealizing or denigrating imaginary that does not adequately define the social "subject" towards which these public policies are directed.

It is indicated, as a relevant factor of the final considerations, that in this sense it is necessary to include demographic variables, gender, historical processes, new types of emerging culture (such as urban tribes), rural-urban differences and family transitions, among others, to accurately redefine this social "subject" and then reach an effective resizing of public policies.

Keywords: social policies, state, youth, neoliberalism.

\section{REFERENCIAS}

ABRAMO, Helena Wendel (org.). Retratos da juventude brasileira, análises de uma pesquisa nacional . Porto Alegre: Editora Fundação Perseu, 2005.

ABRAMOVAY, Miriam. Gangues, galeras, chegados e rappers. Juventude, Violência e Cidadania nas Cidades da Periferia da Brasília. Brasil: Unesco, 1995.

AMBROSI, Nicolás y SANSEVIERO, Rafael. Políticas progresistas de juventud. Experiencias y perspectivas al finalizar el primer gobierno de izquierda.Montevideo: Friedrich-Ebert-Stiftung, 2009.

ANDREWS, Charles. Reificação e Legitimidade. Habermas como Metateoria das Policy Sciences. Universidade de São Paulo- Departamento de Ciência Política, 2003

ANEP (Administración Nacional de la Enseñanza). Unidad Ejecutora de los Programas de Educación Media y Formación Docente (MES y FOD y UTU/BID), Montevideo: ANEP, 2002

ANTUNES, Rircardo. Crisis capitalista contemporánea y las transformaciones en el mundo del trabajo In: Capacitação em serviço social e política social: Modulo 1. Brasilia: CEAD, 1999.

ARAUJO, Ana (org.) Impactos del desempleo. Transformaciones en la subjetividad. Montevideo: Alternativas., 2002

ARAÚJO Fernandes, Antônio y SOUZA BONFIM, Washington. La democratización de la gestión municipal en Brasil: un abordaje teórico del objeto, Argentina: bibliotecavirtual.clacso.org.ar/ar/libros/edicion/diseno/araujo.pdf, 2006.

ASTORI, Danilo. La política económica de la dictadura (EI Uruguay de la Dictadura 1973-1985) Montevideo: Ediciones de la Banda Oriental, 1989.

BANGO, Julio. Políticas de Juventud en América Latina en la Antesala del 2000: Logros, Desafíos y Oportunidades. Madrid: OIJ, 1999. 
BARRÁN, José Pedro y NAHUM, Benjamín. Crisis y radicalización, 1913-1916, tomo 6 de la serie: Batlle, los estancieros y el imperio británico Montevideo: EBO, 1985.

BERENSTEIN, Isidoro. Psicoanálisis de la Estructura Familiar- Del destino a la significación. Argentina: Paidós, 1981.

BÉRTOLA, Luis. Ensayos de historia económica. Uruguay y la región en la economía mundial 1870-1990. Montevideo: Ediciones Trilce, 2000.

BORJA, Jordi. Las ciudades y el planeamiento estratégico. Una reflexión europea y latinoamericana. Caracas: Urbana, 22, 104-117, 1998.

BRESSER PEREYRA, Luiz Carlos y CUNILL GRAU, Nuria. Lo público no estatal en la reforma del Estado. Buenos Aires: Paidós, 1998.

CASTEL, Robert. La metamorfosis de la cuestión social. Una crónica del salariado. Argentina: Paidós, 1997.

CELADE. América Latina: proyecciones de población 1970-2050- Boletín Demográfico 62. Santiago de Chile: Naciones Unidas, 1998.

CELADE-CEPAL-OIJ. Informe 2002. Montevideo: CELADE, 2002.

CEPAL. Panorama Social de América Latina, 1994. Santiago de Chile: Naciones Unidas, 1994.

CEPAL. Panorama Social de América Latina, 1998, Santiago de Chile: Naciones Unidas, 1998.

CEPAL-CELADE-División de Población. Juventud-Población y Desarrollo: problemas, posibilidades y desafíos. Santiago de Chile: Naciones Unidas, 2000.

CEPAL/UNICEF/SECIB. Construir Equidad desde la Infancia y la Adolescencia en Iberoamérica. Santiago de Chile: Naciones Unidas, 2001.

CEPAL. Panorama Social de América Latina, 2000-2001. Santiago de Chile: Naciones Unidas, 2001.

CEPAL. Panorama social de América Latina 2001-2002. Santiago de Chile: Naciones Unidas, 2002.

CEPAL. Juventud, pobreza y desarrollo en América latina y el Caribe., Santo Domingo: Naciones Unidas, 2003.

CINTERFOR. Políticas de juventud en Uruguay. Uruguay: OIT, 1995.

COHENDOZ, Mónica. Identidad joven y consumo: la globalización se ve por MTV. España: http://www.ull.es/publicaciones/latina/a1999coc/35mtv.html, 1999. 
DUFOUR, Dany-Robert. A arte de reduzir as cabeças. Sobre a nova servidão na sociedade ultraliberal. Rio de Janeiro: Companhia de Freud Editora, 2005.

DUSCHATZKY, Silvia y COREA, Cristina. Chicos en banda. Los caminos de la subjetividad en el declive de las instituciones. Buenos Aires: Paidós, 2002.

FORRESTER, Viviane. Una extraña dictadura. Buenos Aires: Fondo de Cultura Económica, 2000.

FRAGA, Paulo Cesar Pontes (Orgs.) Jovens em tempo real. Brasil: DP\&A editora, 2003.

FUNDACIÓN PAZ CIUDADANA. Diagnóstico de la población juvenil en Chile. Santiago, 1999.

FURTADO, Marcelo La trayectoria educativa de los jóvenes: el problema de la deserción. Cuaderno de trabajo $\mathrm{N}^{\mathrm{o}}$ 22, Montevideo: ANEP, 2003.

GOMÁRIZ MORAGA, Enrique. Sistema político y políticas públicas en América Latina http://www.clad.org/portal/publicaciones-del-clad/revista-cladreformademocracia/articulos/038-junio-2007/sistema-politico-y-politicas-publicas-enamerica-latina, 2007

INSTITUTO NACIONAL DE ESTADÍSTICA. Informe 2003 y 2004. Montevideo: http://www.ine.gub.uy/biblioteca/publicaciones.htm , 2004

KLEIN, Alejandro Imágenes del adolescente desde el psicoanálisis y el imaginario social. Condiciones de surgimiento de la adolescencia desde la modernidad y el disciplinamiento adolescentizante desde la pos-modernidad. Montevideo: Psicolibros, 2002

KLEIN, Alejandro.Adolescentes sin Adolescencia: Reflexiones sobre la construcción de subjetividad adolescente bajo el contexto neoliberal. Uruguay: Psicolibro- Universitario, 2006.

KLEIN, Alejandro. Neoliberalismo-Neoevangelismo-Cambios socio-demográficos Posibles marcos epistemológicos frente a algunos desafíos actuales en el campo de las ciencias sociales (los paradigmas ambiguos) Universidad de Zaragoza: Acciones e Investigación en Ciencias Sociales- Escuela Universitaria de Estudios Sociales, 2010.

KYMLICKA, Will y WAYNE, Norman. El retorno del ciudadano. Una revisión de la producción reciente en teoría de la ciudadanía. Madrid: Revista sobre Estado y la Sociedad, 1997.

LAGO, Luciana Corrêa do. A lógica segregadora na metrópole brasileira: novas tesis sobre antigos processos Rio de Janeiro: Cadernos IPPUR/ UFRJ ano XV,No 2, 2002.

MAIER, Henry. Tres teorías sobre el desarrollo del niño. Buenos Aires: Amorrortu, 1980.

MARRERO, Adriana "El futuro del país"-La juventud uruguaya entre el fracaso escolar $\mathbf{y}$ el desempleo. http://www.rau.edu.uy/fcs/soc/curriculums/AMarrero/publicaciones\%20Adriana/Educacion lel\%20futuro\%20del\%20pais.pdf, 2003, 
MINISTERIO DE EDUCACIÓN Y CULTURA. Informe de la Comisión Coordinadora de la Juventud. Montevideo: MEC, 1998.

MOSER, Caroline. La pobreza urbana y la violencia en Jamaica. Washington: Banco Mundial, 1997.

NAHUM, Benjamín. La época batllista (1905-1929). Montevideo: Ediciones de la Banda Oriental, 1975.

O'DONNELL, Guillermo. Pobreza y desigualdad en América Latina: algunas reflexiones políticas. In: TOKMAN, Víctor y O'DONNELL, Guillermo (org.) Pobreza y desigualdad en América latina. Temas y nuevos desafios. Buenos Aires: Paidós, 1999.

OIJ. Políticas de juventud en América Latina: evaluación y reformulación. http://www.cinterfor.org.uy/public/spanish/region/ampro/cinterfor/temas/youth/doc/not/libr o56/libro56.pdf, 1996

ONU. Implementation of the World Programme of Action for Youth to the Year 2000 and Beyond. http://www.un.org/events/youth98/backinfo/ywpa2000.htm, 2001

PANIZZA, Francisco. Uruguay: Batllismo y después- Pacheco, Militares y Tupamaros en las crisis del Uruguay Batllista. Montevideo: Ediciones de la Banda Oriental, 1990.

PILOTTI, Francisco y Camacho, María Claudia. Políticas y programas de juventud en América Latina y el Caribe: Contexto y principales características. Social Protection Discusión Paper Series No 0312 Washington: Banco Mundial, 2003.

PRETECILLE, Edmond. Segregação, Classes e Política na Grande Cidade. Rio de Janeiro: Cuadernos IPPUR/UFRJ vol X No 2, 1996.

RODRÍGUEZ, Ernesto. Políticas públicas de juventud en América Latina: experiencias adquiridas y desafíos a encarar. Madrid: Pensamiento Iberoamericano nº3, 2008.

RODRÍGUEZ, Ernesto. Políticas públicas de juventud en América latina: empoderamiento de los jóvenes, enfoques integrados, gestión moderna y perspectiva generacional. www.urbared.ungs.edu.ar/textos/politicasdejuventud2.pdf, 2003.

SADER, Emir y GENTILI, Pablo (org) La trama del neoliberalismo. Mercado, crisis y exclusión social. Argentina: Universitaria de Bs. As, 1999.

TAVARES, Laura. Os custos sociais do ajuste neoliberal no Brasil. Santiago de Chile: FLACSO, 1999.

TORELLO, Manuel. Educación: Gasto, Desarrollo Humano y Equidad. Documento de Trabajo del Rectorado, No. 23. Montevideo: Universidad de la República, 2004

UNICEF/IBGE. Indicadores sobre criancas e adolescentes. Brasilia: UNICEF, 1997. 
VASCONCELOS, Eduardo y MORGADO, Rossana. Subsídios analíticos e metodológicos para a atuação no Sistema Único de Assistência Social (SUAS), e do Programa de Atendimento Integral à Família. Rio de Janeiro: PAIF/SAS/RJ, 2005.

Trabalho enviado em 10 de março de 2019

Aceito em 30 de janeiro de 2020 